\title{
DESIGN AND CFD ANALYSIS OF A LOW-ALTITUDE VTOL UAV
}

\section{KYOUNG-MOO MIN ${ }^{1}$, FOONG-YI CHIA ${ }^{2} \&$ BONG-HWAN KIM ${ }^{3}$}

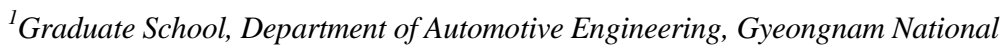

University of Science and Technology, Jinju, Korea

${ }^{2} R \& D$ Center, SAMCO, Sacheon, Korea

${ }^{3}$ Department of Automotive Engineering, Gyeongnam National University

of Science and Technology, Jinju, Korea

\begin{abstract}
In this study, in order to develop a vertical take-off and landing modular body platform that meets take-off and speed performance, each mission file was prepared for conventional take-off (CTOL) and vertical take-off and landing (VTOL) based on the design requirements. The conceptual design took the basic shape and, in the first place, estimated the initial weight of the aircraft components and the entire BOM. After reflecting the components and weights applied to the drones in the design, the main wing shape design, weight center and neutral point prediction, and thrust system design were performed using XFLR5. ANSYS Workbench V14.5 was used for CFD analysis of the aircraft and ICEM-CFD was used as a program to generate Hybrid Mesh (Hexa Mesh \& Prism Mesh). Through analytical techniques, the airfoil used for the main wing was designed as NACA4412 and the tail wing was designed as the V-Tail type, which minimizes the aft effects of the thrust device, and the basic airfoil of the tail wing for stability was selected as NACA0009. Because the droneapplied in this study must have the ability to make both a vertical take-off and a horizontal flight, the N. P. position designed in flight mode and the distance of each rotor of the quadrotor are similarly designed.
\end{abstract}

KEYWORDS: Low-Altitude VTOL UAV, XFLR5, CFD, Aerodynamics Analysis, Airfoil \& Quad-Rotor

Received: Feb 08, 2019; Accepted: Feb 28, 2019; Published: Mar 19, 2019; Paper Id.: IJMPERDAPR201954

\section{INTRODUCTION}

With the recent availability of high-resolution images through drones, the opportunity to utilize these high-quality image materials in the field of cadastral survey is increasing. The use of various reference materials in the field of cadastral measurement increases the need for technology development to guide the cadastral survey performance and performance determination of cadastral measurement engineers. If images are used in the field of cadastral measurement, they can be applied to a variety of tasks, such as pre-survey and data acquisition for non-access areas. If existing aerial photographs are used, the resolution of images (GSD: 20cm) is low, which makes them difficult to identify boundaries. If unmanned aerial vehicles are used for long periods of time, high resolution (within GSD:5cm) video acquisition will be possible for low operating costs, enabling them to be used for such tasks as boundary checking and cadastral inconsistence area investigation. For this purpose, it shall be possible to make a vertical take-off, and the altitude of the vertical take-off shall be increased by at least $25 \mathrm{~m}$, and take-off shall be possible[1,2,3]. In addition, the flight time shall be not less than 20 minutes, and shall be capable of flying with a load of up to $1.5 \mathrm{~kg}$, with a horizontal flight speed of not less than $15 \mathrm{~m} / \mathrm{s}$. In this study, for the purpose of developing a vertical take-off and landing platform of module type drone that meets take-off and airspeed performance, the mission profile such as Figure 1 was prepared based on the above design requirements, 
and the aircraft mission for basic aircraft design was set up for the flight design process. Shape design was carried out in consideration of the above requirements and mission profile, and trade-off study of various drone bodies was carried out. The basic form was taken through concept design, and the most commonly considered part of the consideration was a variety of factors in order to capture the shape in which, a $12 \mathrm{~kg}$ class drone can be flown for more than an hour. Initial weights were estimated from the drone components and from the entire BOM(Bill Of Material) as a whole in the configuration. The design reflected the components and weights applied to the drones, and then performed the design of the main wing shape, the prediction of the center of gravity and neutral point, and the thrust system[4,5].

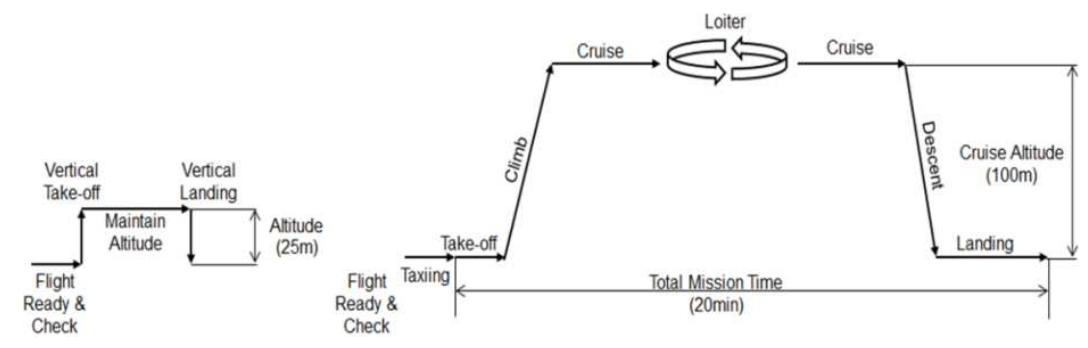

Figure 1 : Mission File of Design Requirement for Low-Altitude Vtol Uav

\section{BASIC DESIGN OF DRONE BODY}

\section{Selection of Airfoil Shape}

Selection of airfoil for successful aircraft flights is very important. In this study, three airfoils (NACA4412, NACA2412, and NACA23012) of the NACA series were selected and compared using XFLR5[6] and Figure 2 represents the shape of three airfoils. For this purpose, lift coefficient, drag coefficient, lift-drag ratio, and pitching moment coefficient were selected by comparing the speed of operation of unmanned aircraft, the Reynolds number of 500,000 corresponding to the altitude area and Figure 3 is a graph comparing the characteristics of each airfoil. Analyzing the first and second drag coefficient graphs, the airfoil of NACA4412 has the largest lift coefficient and has the softest stall characteristics. In addition, a comparative analysis of the positive ratio for each airfoil indicated that the positive ratio of the NACA4412 airfoil is better than the NACA212 and NACA23012 airfoil. And the third pitching moment coefficient graph shows that the NACA4412 airfoil is the most stable of the three airfoils because it has a smooth pitching moment characteristic of the airfoil and the pitching moment coefficient is negative in the various angle of attack(AoA), where the aircraft will operate. Therefore, the airfoil used for main wing is selected as NACA4412.

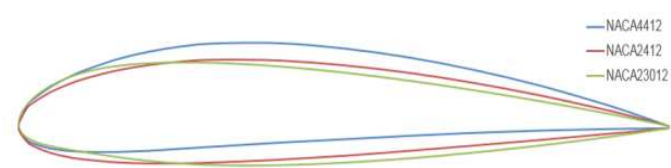

Figure 2: Configuration of Airfoil 


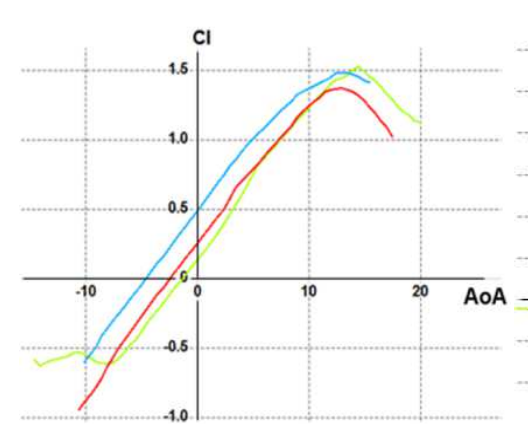

(a) Clvs. AoA

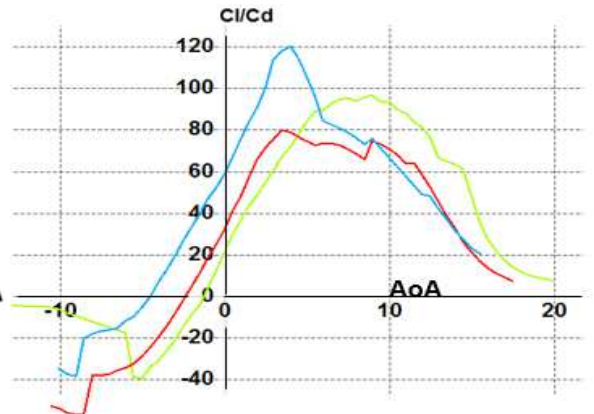

(b) $\mathrm{Cl} / \mathrm{Cd}$ vs. AoA

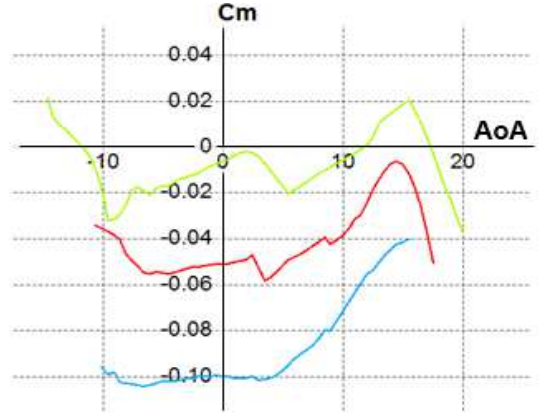

(c) Cm vs. AoA

Figure 3: Lift, Drag and Pitching Moment Coefficients with AoA

\section{Dimension of Airfoil}

The maximum take-off weight of the drone was estimated to be about $12 \mathrm{~kg}$. The lift (L) and the mass (W) of the drone are the same under horizontal flight, the area of the wing can be calculated in the following equation (1).

$$
W=L=\frac{1}{2} \rho V_{\text {cruise }}^{2} S_{\text {wing }} C_{L}
$$

Since the operating area of the drone developed is operated at low altitudes below $200 \mathrm{~m}, \rho\left(1.225 \mathrm{~kg} / \mathrm{m}^{3}\right)$ is assumed to be the air density at sea level altitude, and the lift coefficient $\left(C_{L}\right)$ was calculated as $C_{L}=0.5$ by data from the NACA4412 airfoil. It is also assumed that $V_{\text {cruise }}$ is the cruising speed of a typical drone, $20 \mathrm{~m} / \mathrm{s}$. As a result, the calculated wing area $\left(S_{\text {wing }}\right)$ is $0.96 \mathrm{~m}^{2}$ and the two-dimensional plane form of main wing is designed as shown in Figure 4 . In the design section on tail wing shape and location, the push type thrust system was selected for efficient operation of cameras and mission equipment. Therefore, the tail wing was designed as a V-tail type with minimal influence of wake flow over the thrust device, and the basic airfoil of the tail wing for stability was selected as NACA0009 which is a symmetrical shape without camber and is small in thickness ratio, making it easy to manufacture. The vertical position of the V-tail is designed $100 \mathrm{~mm}$ below the base of the root chord on the main wing to minimize the effects of the propeller and wake flow of motor. In addition, the angle of incidence of the $\mathrm{V}$-tail was designed at $-2^{\circ}$ to minimize elevator trim during cruise flight. Figure 5 represents the three-dimensional shape of the main and tail wings, while Figure 7 represents the position of the main and tail wings.

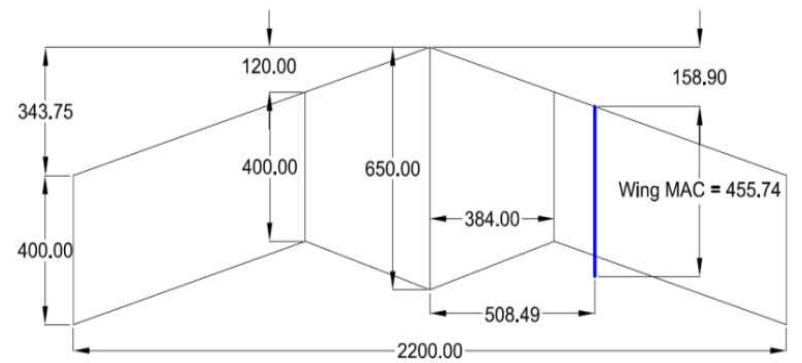

Figure 4: Shape and Dimension of Main Wing

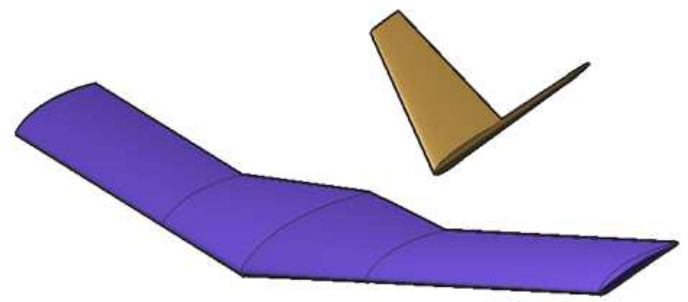

Figure 5: 3D Configuration of Main and Tail Wing

\section{Center of Gravity and Neutral Point}

The center of gravity (C. G) and the neutral point (N. P) depend on the size and position of the tail wing. Thus, through the XFLR5 program using the Panel Method, the force and moments caused by air force between the main and tail wings were briefly calculated. For the calculation of the neutral point of an aircraft, the aircraft weight was set to an initial 
estimate of $12 \mathrm{~kg}$, and the speed of drone was set at $20 \mathrm{~m} / \mathrm{s}$. The neutral point is the point at which the moment changes with the angle of attack is zero. From this point, the static margin (S. M) varies the position of the C. G to the $10 \%$ point, simulating the change of the moment for the angle of attack. In the simulation result shown in Figure 6, the neutral point was calculated as a point $320 \mathrm{~mm}$ from the root chord leading edge of the main wing, and the center of gravity was determined as shown in Figure 7, when calculated as $10 \%$ of the margin.

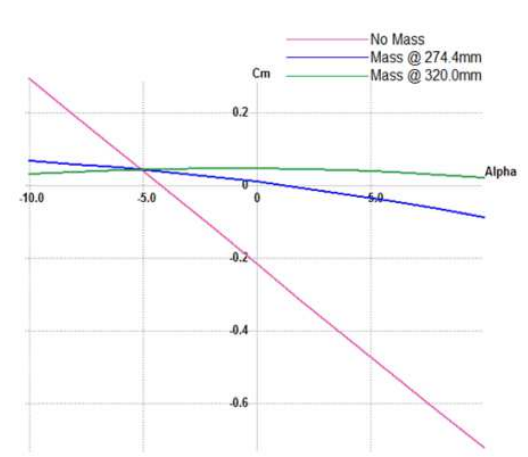

Figure 6: Moment Variation by Change of Center of Gravity

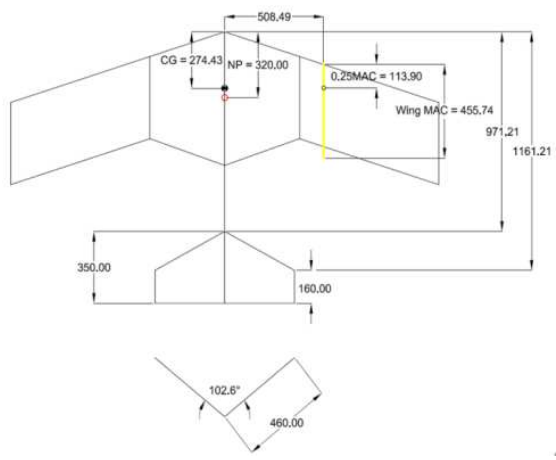

Figure 7: Center of Gravity and Neutral Point of Main Wing

The position of the quad-rotor motor may vary depending on the C. G position of the drone. The drone that are applied in this study should have the ability to make both a vertical takeoff and a horizontal flight. It is therefore important to design N. P. positions and the distance of each quad-rotor. Figure 8 indicates the position of the quad-rotor satisfying the above conditions.

\section{CFD ANAYSIS OF DRONE BODY}

\section{Two Cases of CFD Analysis Model}

In order to identify the aerodynamic characteristics, a CFD analysis of the entire drone system was performed on two cases: The first case was carried out without forward thrust motors and quad-rotor system, and the second case examined how the overall aerodynamic characteristics of the drone were changed with forward thrust motors and quad-rotor systems similar to the actual shape[7,8,9].

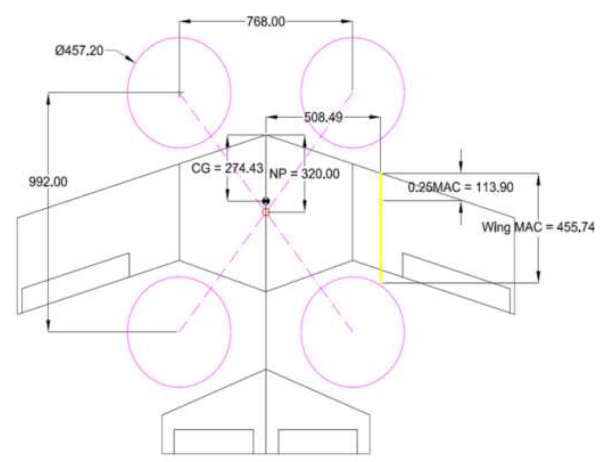

Figure 8: Position of Quad-Rotor 


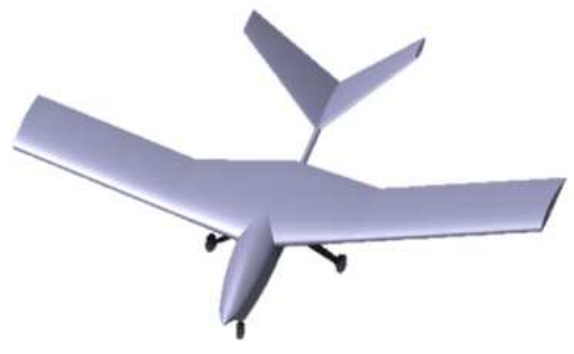

(a) Case 1: without Propulsion System

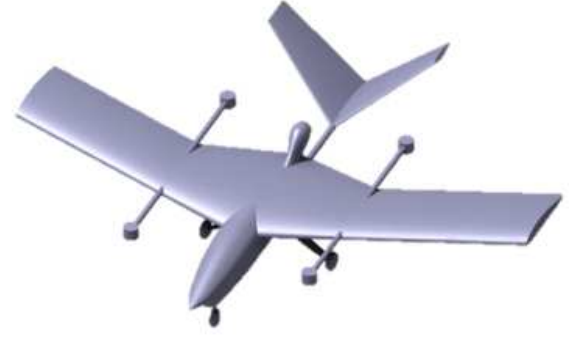

(b) Case 2: with Propulsion System

Figure 9: Two Cases for CFD Analysis

\section{CFD Analysis Method}

ANSYS Workbench V14.5 was used for CFD analysis, and Hybrid Mesh (Hexa Mesh \& Prism Mesh) was applied using ICEM-CFD for grid generation. The boundary layer height y+ value near the wall was set to 1, and prism mesh was used and calculated at approximately 2 million grid points as shown in Figure 10. Figure 11 represents the y+ distribution applied to the shape of a drone. FLUENT was used for CFD analysis, and the inlet conditions were varied by angle attack which is from $-8^{\circ}$ to $16^{\circ}$ at the drone speed from $20 \mathrm{~m} / \mathrm{s}$. 2nd upwind k-omega SST model was applied to the Turbulence Model [10].

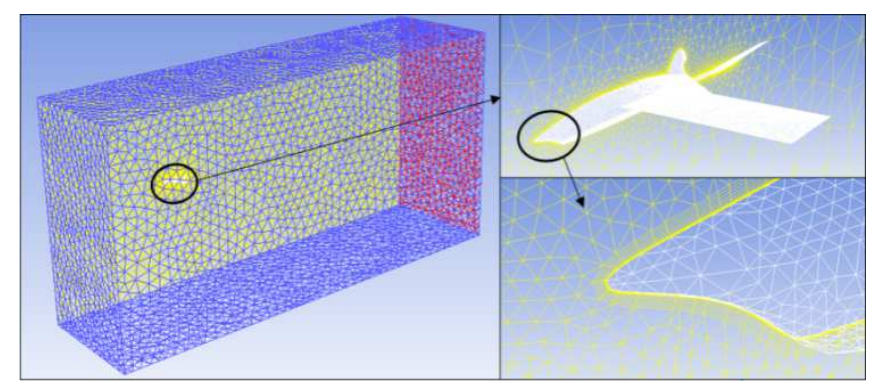

Figure 10: Domain and Grid Generation by Hybrid Meshing

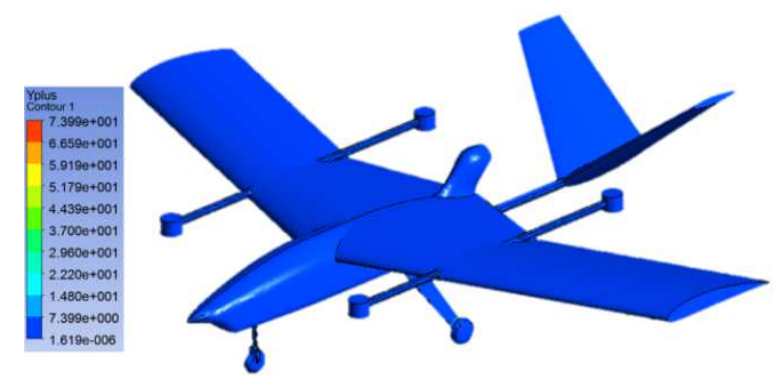

Figure 11: y+ Contour of VTOL UAV for CFD Analysis

Figure 12 shows the characteristics of the variation in lift and drag coefficient according to the angle of attack for the two cases. The analysis results showed that drag does not significantly affect the presence or absence of a quad-rotor system in the range of $-5^{\circ}$ to $5^{\circ}$, whereas the overall lift was reduced in Case 2[11,12]. At angle of attack more than $10^{\circ}$, the drag can be seen to increase significantly. It can also be seen that, regardless of the case, the angle of stall is about $14^{\circ}$. It was confirmed that $\mathrm{C}_{\mathrm{Lmax}}$ (maximum lift coefficient) decreased from 0.52 to 0.48 and $\mathrm{C}_{\mathrm{L} 0}$ (lift coefficient at angle of attack $0^{\circ}$ ) decreased from 0.19 to 0.16 . As can be seen from the pressure distribution which is shown in Figure 13, there is a large loss of pressure in Case 2 equipped with quad-rotor system and thrust motor device. 

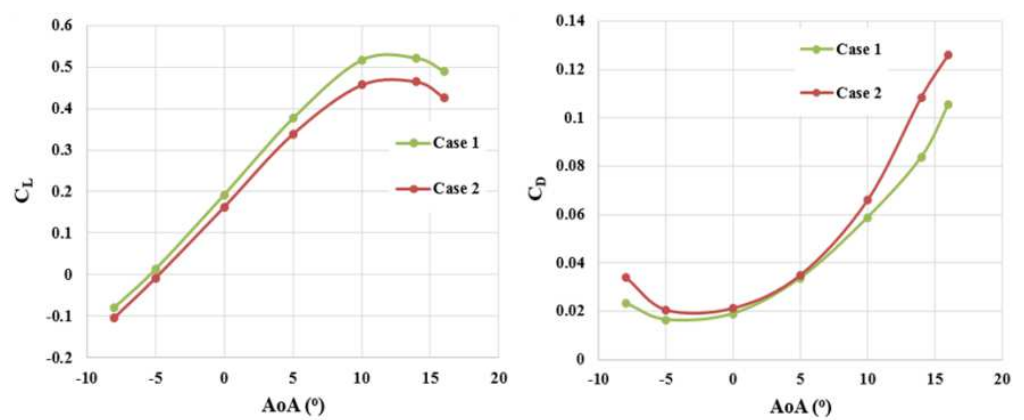

Figure 12: Lift and Drag Coefficient Distribution with Angle of Attack

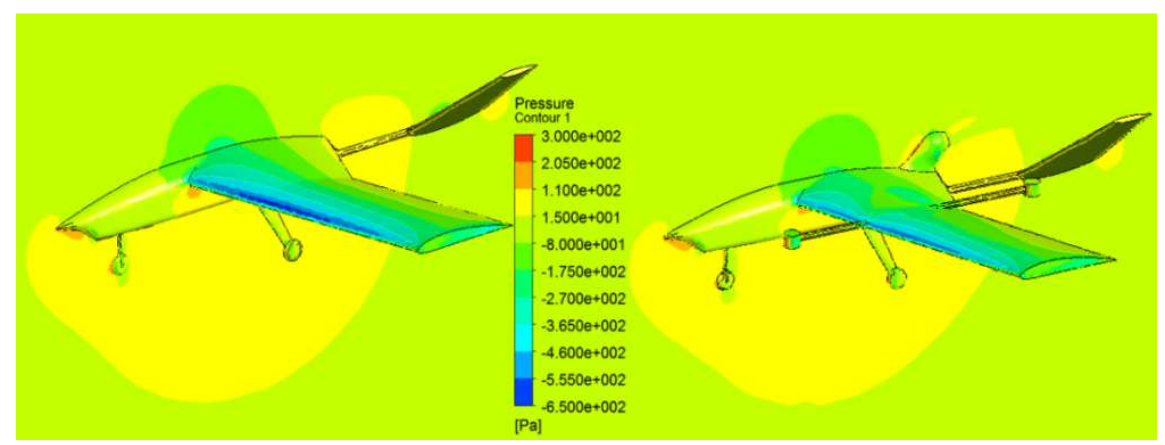

(a) Case1

(b) Case2

Figure 13: Pressure Distribution of 2 Cases at $\mathrm{AoA}=10^{\circ}$

Velocity vector and streamline distributions are shown in Figure 14 and Figure 15, and there is a large presence of pressure gradient behind the motor mount. As shown by Figure 14 and Figure 15, the effect of vortex flow above and below wings produced in quad-rotor systems results in a large loss of lift. Figure 16 illustrates the formation of vortex shedding by wing tip and quad-rotor systems at an angle of attack $10^{\circ}$.

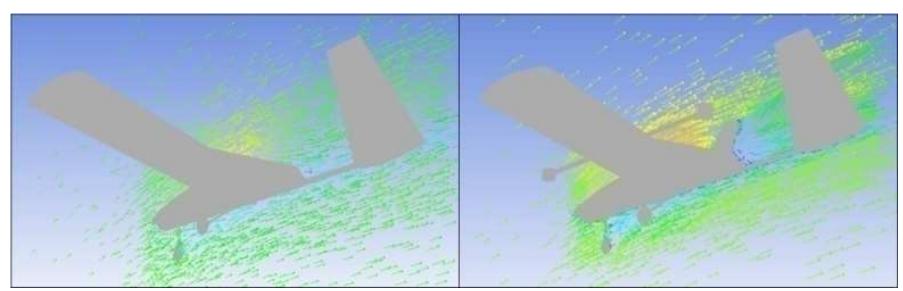

Figure 14: Velocity Vector Distribution of 2 Cases at $\mathrm{AoA}=10^{\circ}$

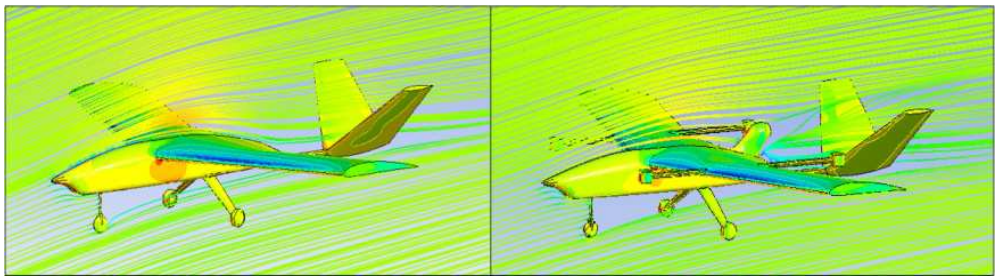

Figure 15: Streamline Distribution of 2 Cases at $\mathrm{AoA}=10^{\circ}$ 


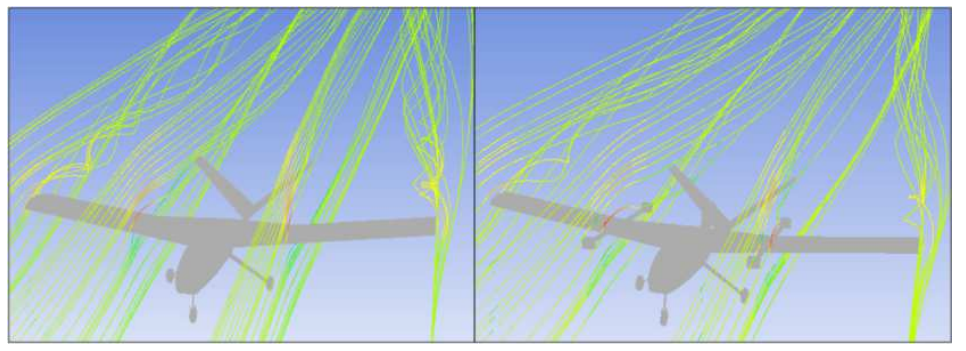

Figure 16: Vortex Formation of Wings at $\mathrm{AoA}=10^{\circ}$

\section{CONCLUSIONS}

The low altitude vertical take-off and landing UAVs, which combine airplane wings and quad-rotors, are very complicated, so the design of UAVs requires thorough and careful consideration. Therefore, XFRL5 was used for air force design and ANSYS Workbench V14.5 was used for aerodynmics performance evaluation. Because the drone applied in this study must have the ability to make both a vertical take-off and a horizontal flight, the N. P position designed in aircraft mode and the distance of each rotor of the quad-rotor. The main wing is selected as the most stable NACA4412 with soft moment characteristic of the airfoil, and the tail wing is designed as the V-tail type, which minimizes the wake effects of the thrust device, and the basic airfoil of the tail wing for stability is a symmetrical shape without camber, and is easy to manufacture. The vertical position of the V-tail is designed $100 \mathrm{~mm}$ below the base of the root chord on the main wing to minimize the wake effects of the propeller and motor.

\section{REFERENCES}

1. IAI Panther, 2014, http://www.iai.co.il/2013/35673-41636-en/IAI.aspx.

2. Ozdemir, U. et al, 2014, "Design of a Commercial Hybrid VTOL UAV System, Journal of Intelligent and Robotic Systems", 74(1-2), pp. 371-393.

3. S. Verling, J. Zilly, 2013, "Modeling and Control of a VTOL Glider", Bachelor thesis, ETH, Zurich.

4. Armutcuoglu, O., 2000, "The Conceptual Design Of a Tilt-Duct VTOL UAV", M. Sc. Thesis, Middle East Technical University.

5. Jon Ahn, 2011, "Conceptual Design and Performance Analysis of a Solar Powered HALE UAV Considering Practical Constraints", Journal of the Korean Society for Aeronautical and Space Sciences, pp. 379-385.

6. Nikam, Harshal., Mishra, Prem., \& Bharambe, Sayali. (2014). Design and Analysis of Brake Rotor with Parameter Optimization. International Journal of Automobile Engineering Research and Development (IJAuERD), 4(4), 21-30.

7. XFLR5: http://www.xflr5.com/xflr5.htm

8. Theresa Shafer et al., 2014, AIAA SciTech, "Comparison of Computational Approaches for Rapid Aerodynamic Assessment of Small UAVs", http://arc.aiaa.org/doi/abs/10.2514/6.2014-0039

9. B. Ravi Theja, Dr. M. Satyanarayana Gupta, "Design ad Fluid Flow Analysis of Unmanned Aerial Vehicle (UAV)", International Journal of Science and Research (IJSR) ISSN (Online): 2319-7064.

10. Dumitru Pepelea, Marius Gabriel Cojocaru, Adrian Toader, Mihai Leonida Niculescu, 2016, "CFD Analysisfor UAV of Flying Wing", Scientific Research and Education in the Air Forec-Afases 2016.

11. Sravan Kumar Khuntia, Amandeep Singh Ahuja, 2018, "Optimumal Designand CFD Analysisof Wingof a Small-scale UAV to Obtain Maximum Efficiency", International Journal of Computer Application (2250-1797) Issue 8 Volume 1. 
12. Saboo, S., \& Gupta, C. P. (2013). Design Of Photonic Crystal Fiber For Minimum Confinement Loss By Varying The Size Of Holes. International Journal of Electronics and Communication Engineering (IJECE), 2(2).

13. Karthik M A, Srinivasan K, Srujan S, Subhash Holla H S, Suraj Jain M., 2017, "Design and CFD Analysis of a Fixed Wing for an Unmanned Aerial Vehicle", International Journal of Latest Engineering Research and Applications (IJLERA) ISSN: 24557137, 02(07), pp. 77-85.

14. S. H. Park, K. Chang, H. J. Shim, D. J. Sheen and S. H. Park, 2016, "Computational Fluid Dynamics of the Low-speed Longitudinal Aerodynamic Characteristics for BWBType UCAV Configuration", Journal of Computational Fluids Engineering, 21(3), pp. 48-54. 